\title{
The nucleotide addition cycle of RNA polymerase is controlled by two molecular hinges in the Bridge Helix domain
}

\author{
Robert OJ Weinzierl
}

\begin{abstract}
Background: Cellular RNA polymerases (RNAPs) are complex molecular machines that combine catalysis with concerted conformational changes in the active center. Previous work showed that kinking of a hinge region near the $\mathrm{C}$-terminus of the Bridge Helix $\left(\mathrm{BH}-\mathrm{H}_{\mathrm{C}}\right)$ plays a critical role in controlling the catalytic rate.

Results: Here, new evidence for the existence of an additional hinge region in the amino-terminal portion of the Bridge Helix domain $\left(\mathrm{BH}-\mathrm{H}_{\mathrm{N}}\right)$ is presented. The nanomechanical properties of $\mathrm{BH}-\mathrm{H}_{\mathrm{N}}$ emerge as a direct consequence of the highly conserved primary amino acid sequence. Mutations that are predicted to influence its flexibility cause corresponding changes in the rate of the nucleotide addition cycle (NAC). $\mathrm{BH}-\mathrm{H}_{\mathrm{N}}$ displays functional properties that are distinct from $\mathrm{BH}-\mathrm{H}_{\mathrm{C}}$, suggesting that conformational changes in the Bridge Helix control the NAC via two independent mechanisms.
\end{abstract}

Conclusions: The properties of two distinct molecular hinges in the Bridge Helix of RNAP determine the functional contribution of this domain to key stages of the NAC by coordinating conformational changes in surrounding domains.

\section{Background}

RNA polymerases (RNAPs) play a central role in the regulation of gene expression. Like the majority of the enzymes involved in fundamental biological information-processing functions (for example, replication, transcription, recombination, repair), RNAPs are probably best viewed as intricate molecular machines. The movement of nucleic acid substrates, coupled with various types of active site chemistries, requires a precisely orchestrated sequence of conformational changes of protein domains during the transcription cycle (for recent reviews see [1-4]).

The nanomechanical mechanisms guiding the structural rearrangements of domains within the active site are still very poorly understood. Thus far, models of the fundamental reaction catalyzed by RNAPs, the nucleotide addition cycle (NAC), have predominantly been derived from a series of crystal structures that contain RNAPs as apoenzymes (for example [5-9]), or complexed with various

Correspondence: r.weinzierl@imperial.ac.uk

Department of Life Sciences, Imperial College London, London SW7 2AZ, UK substrates and inhibitors (for example [10-15]). Such structures, revealing (among other features) pre- and posttranslocation states of RNAPs, have provided the basis for various hypotheses concerning the molecular mechanism of the NAC [1-4,16,17]. There are, however, two potential shortcomings associated with such approaches. First, in order to 'freeze' the RNAPs in a crystallizable conformation, substrate analogs or inhibitors need to be chosen that stop the reaction cycle at a specific point. This may result in the adoption of 'off-pathway' conformations that do not represent normal enzyme states. A second, more fundamental, problem is that short-lived intermediate structures cannot be captured in crystals because they are thermodynamically or kinetically unstable. Yet, it is likely that an awareness of the existence and functional significance of such intermediates will be required to develop a deeper understanding of the mechanisms operating within molecular machines.

We have designed new experimental tools to complement ongoing structural investigations. Based on the ability to assemble an active RNAPII-like enzyme from recombinant subunits in vitro, it is possible to modify 
any residue within an intact RNAP to introduce a variety of targeted mutations into any functional domain participating in the NAC [18]. Such a strategy not only allows specific predictions based on available X-ray structures to be tested, but also can be used to explore systematically the functional contributions of individual domains to biochemically detectable activities. New robotic methods that facilitate the labor-intense highthroughput mutagenesis/assembly steps and transcription assays allow this approach to be implemented on a large scale [19]. In a recent study we systematically replaced each of 17 consecutive residues of the Bridge Helix domain with all other 19 possible amino acid side chains [20]. The Bridge Helix, a 35 amino acid $\alpha$-helix spanning the RNAP active site, controls the flow of nucleic acid substrates and nucleotide precursors through the catalytic site (Figure 1A). Some of the earliest models of the NAC were based on the observation that the $\mathrm{C}$-terminal portion of the Bridge Helix was kinked in some X-ray crystal structures of bacterial
RNAPs $[5,8]$, but appeared straight in numerous other crystals of bacterial, archaeal and eukaryotic RNAPs. The existence of two alternative Bridge Helix conformations made it seem likely that a periodic oscillation between straight and kinked conformations would be implicated in translocating RNAPs in single base-pair steps along the DNA template strand [7,10,21-24]. High-throughput mutagenesis of the C-terminal portion of the Bridge Helix provided unexpectedly clear evidence in support of Bridge Helix kinking; mutations destabilizing the normal $\alpha$-helical conformation in certain positions cause a substantial increase in the specific activity of RNAPs. This phenomenon, referred to as superactivity, relieves a constraint on the catalytic mechanism by increasing the frequency of Bridge Helix isomerization between straight and kinked conformations $[20,25]$.

While the combination of structural observations and mutagenesis data clearly highlights the functional contribution of the $\mathrm{C}$-terminal portion of the Bridge Helix

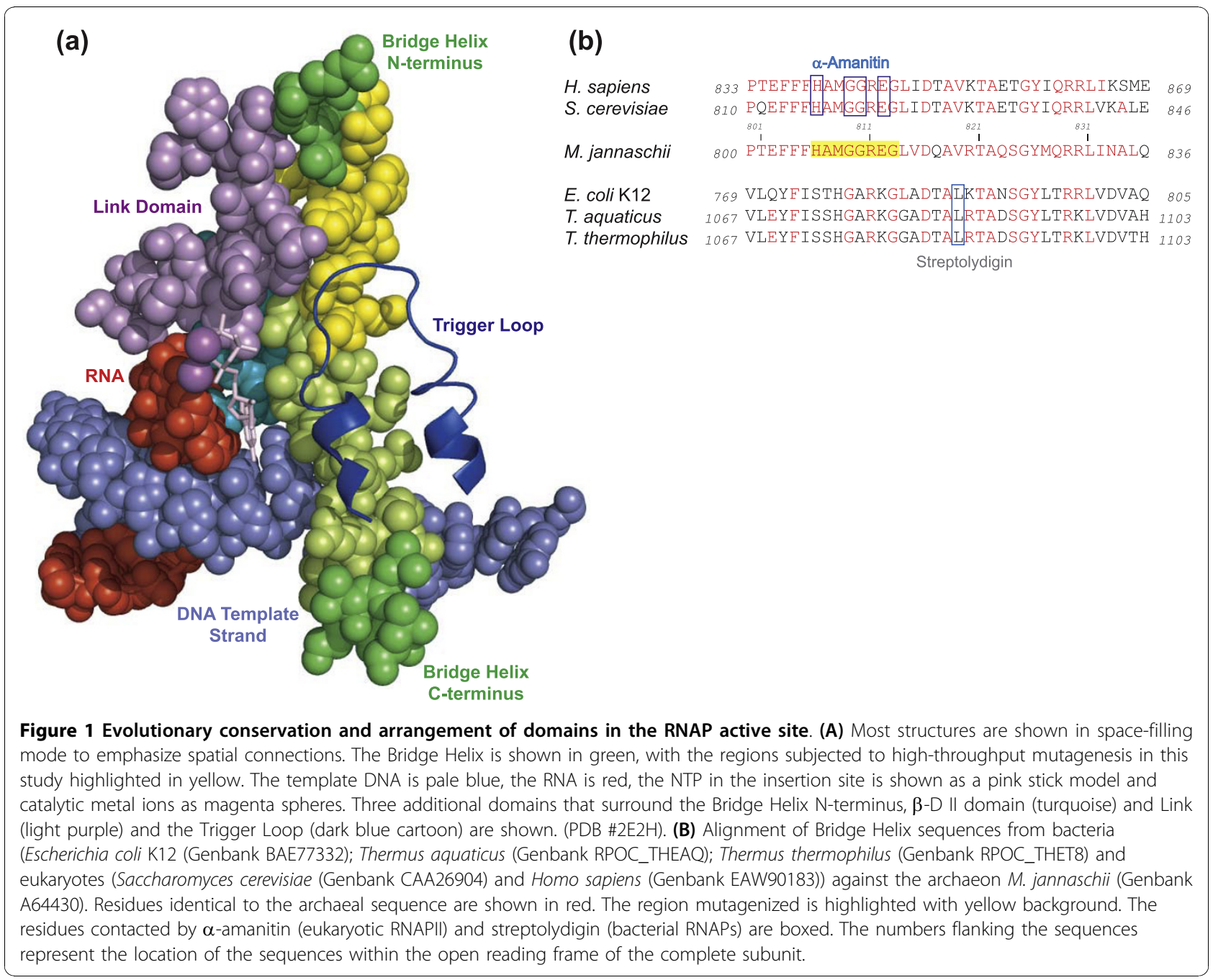


towards controlling the rate of the NAC, the role of the $\mathrm{N}$-terminal portion of the Bridge Helix has thus far remained enigmatic. The primary sequence of this region is exceptionally highly conserved during evolution; for example, the sequences of the N-terminal 15 amino acids are identical between the archaeon Methanocaldococcus jannaschii and humans, and differ by only a single residue from yeast (Figure 1B). Such a high degree of structural conservation over more than two billion years of evolution can be partially accounted for by the fact that the Bridge Helix $\mathrm{N}$-terminus is tightly surrounded by other domains and may therefore be spatially and evolutionarily constrained due to the need to maintain an extensive network of protein-protein interactions (Figure 1A; Additional files 1, 2 and 3; $[12,15,25,26])$. In apparent agreement with this view, all available X-ray structures of RNAPs show the N-terminal portion of the Bridge Helix in a rigidly $\alpha$-helical conformation, suggesting the absence of significant conformational changes. For this reason, none of the current models of RNAP function consider the Bridge Helix N-terminus to play any dynamic role during the NAC [1-4,16,17].

New evidence presented here, based on a combination of high-throughput mutagenesis studies and molecular dynamics simulations, demonstrates that such a static view of the Bridge Helix N-terminus is untenable. The results show that this region contains a highly localized molecular hinge, and that the conformation of this site has a substantial influence on the rate of the NAC. In combination with the previously identified C-terminal hinge region, the data reinforces the overarching concept that the Bridge Helix plays a predominantly nanomechanical role during the translocation stage of the NAC by coordinating conformational changes in surrounding domains.

\section{Results}

\section{High-throughput mutagenesis reveals evidence for an} $\mathrm{N}$-terminal hinge

The N-terminus of the Bridge Helix of the RNAP from the euryarchaeon Methanocaldococcus jannaschii was dissected by high-throughput mutagenesis [19,20]. In this automated approach, each amino acid within the target region (Figure 1B) was replaced with all other 19 residues to reveal local structural requirements. The mutants were then assayed in robotic promoter-independent transcription assays, which provide a consistent measure of the synthetic rate of the NAC and correlate directly with results obtained from a variety of promoter-dependent, abortive- and elongation transcription assays ([18-20]; S. Wiesler and ROJW, unpublished data).

The results reveal unexpected insights into the function of the Bridge Helix N-terminus (Figure 2A). The most eye-catching phenotypes are associated with the $m j \mathrm{~A}^{\prime}$ M808 position, which constitutes - both qualitatively and quantitatively - a hotspot for superactivity caused by a chemically diverse range of substitutions. This suggests that the M808 position is structurally very delicately balanced and substitutions with more hydrophilic, bulky hydrophobic, and/or charged residues cause a substantial local disturbance. The degree of superactivity in the strongest N-terminal substitution ( $m j \mathrm{~A}^{\prime} \mathrm{M} 808-\mathrm{P}$; $>240 \%$ superactivity) greatly exceeds the highest level of superactivity displayed by the strongest $\mathrm{C}$-terminal mutant (mjA' S824-P; approximately $170 \%$ superactivity; Figure 2A). The observation that the replacement of M808 with proline results in the highest increase in specific activity immediately suggests that the phenotypes are caused predominantly by disruption of the local secondary structure; proline residues destabilize $\alpha$-helices due to a lack of hydrogen bonding and steric interference with the backbone of the preceding turn [27]. In a manner highly reminiscent of the previously characterized mjA' S824-P substitution phenotype, proline substitutions of the residues immediately surrounding mjA' M808 cause a sharp drop in RNAP catalytic activity, emphasizing the highly localized effect of such conformationally induced changes (Figure 2B; [20]). The locations of two functionally acceptable proline substitutions thus mark the presence of two discrete and separate molecular hinges, that will subsequently be referred to as $\mathrm{BH}-\mathrm{H}_{\mathrm{N}}$ ('Bridge Helix - Hinge N-terminal'; typified by $m j \mathrm{~A}^{\prime} \mathrm{M} 808-\mathrm{P}$ ) and $\mathrm{BH}-\mathrm{H}_{\mathrm{C}}$ ('Bridge Helix - Hinge $C_{\text {-terminal }}$; typified by $m j \mathrm{~A}^{\prime}$ S824-P). The shared functional property of M808-P and S824-P substitutions, causing maximal levels of superactivity, emphasizes that increased Bridge Helix kinking at the two hinges correlates directly with an increased rate of nucleotide addition [20,25].

Extensive database searches, covering completed genome sequences of a large variety of pro- and eukaryotic species, show that naturally occurring proline substitutions in the Bridge Helix primary sequences are exceptionally rare. The only known instances of proline residues occurring naturally anywhere in the $\mathrm{N}$-terminal part of the Bridge Helix are found in the bacteria Orientia tsutsugamushi $[28,29]$, and certain isolates of Arcobacter butzleri [30] and Bacillus subtilis [31]. In each case, the substituted position is precisely orthologous to $m j \mathrm{~A}^{\prime} \mathrm{M} 808$ (Figure 2C). In the C-terminal half of the Bridge Helix, the highly divergent plant RNAPIV and RNAPV enzymes display a strong tendency for a proline residue at the position orthologous to archaeal S824 (Figure 2C; [32,33]).

\section{Molecular dynamics reveal a structural basis for the $\mathrm{BH}$ - $\mathrm{H}_{\mathrm{N}}$ hinge mechanism}

Insights into the conformational changes of the $\mathrm{BH}-\mathrm{H}_{\mathrm{C}}$ are based on certain $\mathrm{X}$-ray structures of bacterial 


\section{(a)}

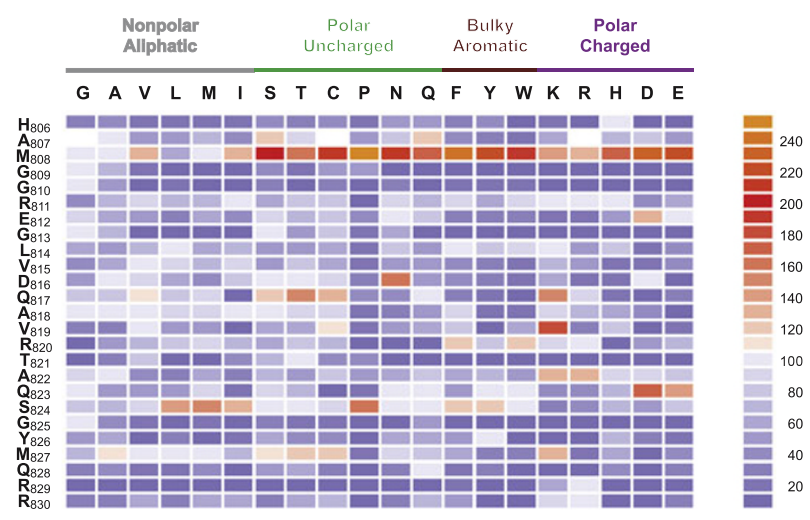

(b)

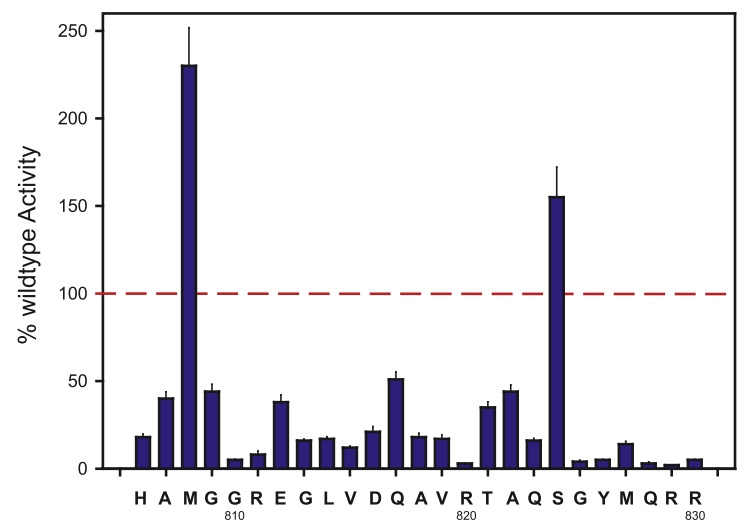

(c)

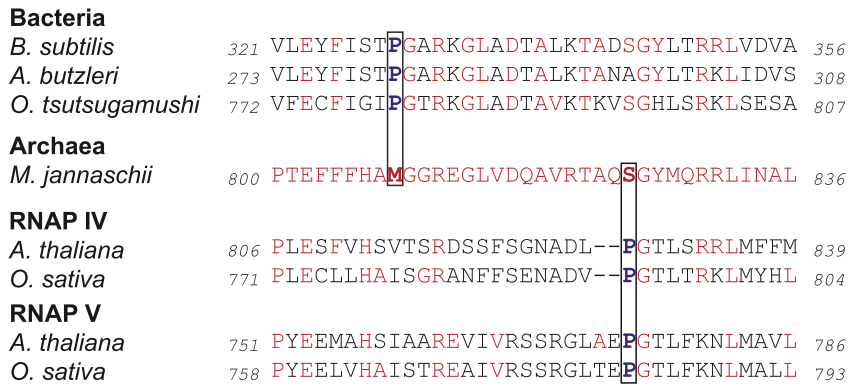

Figure 2 High-throughput mutagenesis of the Bridge Helix. (A) The specific activities in recruitment-independent transcription assays of systematic substitutions of archaeal Bridge Helix residues $\mathrm{H} 806$ to $\mathrm{R} 830$ are shown as a heat map relative to the activity of the wildtype enzyme (see adjacent scale for comparison). Substitutions in M808 cause an exceptional increase in catalytic activity with chemically diverse side chains. Previously published data (L814 to R830; [20]) are included to provide context. All assays were performed in at least quadruplicate with standard deviations within $12 \%$ of the average value. (B) Plot of proline substitutions across the Bridge Helix. The wildtype activity level (100\%) is marked with a dashed red line. The substituted residues in the M. jannaschii Bridge Helix are shown along the horizontal axis. Most proline substitutions cause a severe reduction in the specific activity, except at positions M808 and S824, where proline substitutions cause superactivity. All assays were performed in at least quadruplicate, with error bars showing standard deviation from the average value. (C) Naturally occurring prolinesubstitutions (highlighted in boxes). The Bridge Helix sequences of three bacterial species, Orientia tsutsugamushi (Genbank YP_001248195 (Boryong)/YP_001938485 (Ikeda)), isolates of Arcobacter butzleri (Genbank AAZ80810) and Bacillus subtilis (Genbank BAA10999), as well as representative examples of plant RNAP IV and V Bridge Helix sequences from Arabidopsis thaliana (Genbank AAY89363 and NP_181532, respectively) and Oryza sativa (Genbank EEE70198 and EEE56320, respectively) are aligned against the M. jannaschii sequence (Genbank A64430). The bacterial sequences each contain a single proline residue corresponding to mjA' M808, whereas proline substitutions in RNAP IV and V align with $m j A^{\prime}$ S824. Residues identical to the archaeal sequence are shown in red. 
RNAPs that were fortuitously crystallized in a kinked Bridge Helix conformation $[5,8]$. Kinking of $\mathrm{BH}-\mathrm{H}_{\mathrm{C}}$ is stabilized by intramolecular interactions between amino acid side chains flanking each side of the hinge ([8,20]; Heindl et al., unpublished observations). The location of the $\mathrm{BH}-\mathrm{H}_{\mathrm{C}}$ and its kinking properties are thus predetermined by the local amino acid sequence. This raises the question as to whether intrinsic structural features could also account for the molecular mechanism underlying $\mathrm{BH}-\mathrm{H}_{\mathrm{N}}$ function. Inspection of the primary sequence surrounding $\mathrm{BH}-\mathrm{H}_{\mathrm{N}}$, coupled with insights obtained from molecular dynamic (MD) simulations [34-36], provide a plausible explanation for the structural basis of the $\mathrm{BH}-\mathrm{H}_{\mathrm{N}}$ hinge. Systematic sampling of conformational states at five picoseconds (ps) intervals in a series of 27 independent 200 ps semi-quantitative MD simulations highlights the presence of distinct areas prone to local unfolding, with the most prominent peak centered around mjA' G810 (Figure 3A). Under these simulation conditions, most other regions of the Bridge Helix, including the $\mathrm{N}$ - and $\mathrm{C}$-termini, maintain their $\alpha$ helical conformations at all stages throughout the simulations. Closer inspection of the simulated structures formed by $\mathrm{BH}-\mathrm{H}_{\mathrm{N}}$ kinking reveals a molecular switching mechanism that can be rationalized directly on the basis of the primary amino acid sequence and $\alpha$-helical geometry (Figure $3 \mathrm{~B}$ ). The kinking of $\mathrm{BH}-\mathrm{H}_{\mathrm{N}}$ critically involves the two glycine residues G809 and G810 that are located immediately C-terminal to M808 and are essentially invariant in all archaeal and eukaryotic RNAPIIs (Figure 1B). Glycine residues display low helixforming propensity because their high conformational flexibility is entropically unfavourable within geometrically constrained $\alpha$-helical structures $[37,38]$. This increased flexibility allows G809 and G810 to flip out of the $\alpha$-helical conformation to create a flexible hinge (Figure 3B). The flipped conformation is then stabilized further through a variety of non-covalent interactions of M808 with R811 and E812 (Figure 3C). The kinking model is based on elementary structural and thermodynamic principles (as represented by the MD force field), but also strongly supported by the phenotypes of the G809 and G810 mutagenesis series (Figure 2A). In both positions, any residue other than glycine causes a severe reduction in the catalytic activity of RNAP due to an increased helix propensity, which reduces the likelihood of $\mathrm{BH}-\mathrm{H}_{\mathrm{N}}$ kinking. In contrast, another glycine residue located slightly more C-terminal, G813, is noticeably less sensitive to change (Figure 2A). Similarly, the stabilization of $\mathrm{BH}-\mathrm{H}_{\mathrm{N}}$ through van der Waals contacts between M808 and R811 and E812 appears to be relatively non-specific, so that a chemically diverse group of side chains are either acceptable (especially in the R811 position), or result in enhanced stabilization of the
$\mathrm{BH}-\mathrm{H}_{\mathrm{N}}$ kink (M808 substitution series). The fundamental requirement for a large side-chain in the M808 position is particularly evident from the fact that certain amino acids with smaller groups $(\mathrm{G}, \mathrm{A})$ are not capable of causing the superactivity associated with enhanced $\mathrm{BH}-\mathrm{H}_{\mathrm{N}}$ kinking. It is therefore apparent that the molecular properties of $\mathrm{BH}-\mathrm{H}_{\mathrm{N}}$, such as its structural stability, are directly determined by the primary amino acid sequence and emerge spontaneously from MD simulations as a property inherent in the primary sequence of the Bridge Helix domain. Mutagenesis changes the biophysical properties of $\mathrm{BH}-\mathrm{H}_{\mathrm{N}}$, which is, in turn, directly reflected in altered NAC rates (Figure 2A).

\section{$\mathrm{BH}-\mathrm{H}_{\mathrm{N}}$ and $\mathrm{BH}-\mathrm{H}_{\mathrm{C}}$ operate in environments with widely different structural constraints}

The presence of two distinct hinges in the Bridge Helix raises the question whether $\mathrm{BH}-\mathrm{H}_{\mathrm{N}}$ and $\mathrm{BH}-\mathrm{H}_{\mathrm{C}}$ are involved in the same mechanism during the NAC. Kinking of either of the two hinges will result in considerable local distortions, predicted to include a spatial redeployment of amino acid side chains and changes in the overall length, flexibility and general topology of the Bridge Helix domain. Kinking of $\mathrm{BH}-\mathrm{H}_{\mathrm{N}}$ could result in altered interactions with adjacent domains, such as the $\beta \mathrm{D}-\mathrm{II}$, Link and F-Loop domains (Figure 1A; Additional files 1, 2 and 3 ), whereas hinge movements in $\mathrm{BH}-\mathrm{H}_{\mathrm{C}}$ are expected to affect the position and/or mobility of the DNA-RNA hybrid and Trigger Loop conformation (Figure 1A; [20]).

Intriguingly, RNAP IV Bridge Helices also contain an additional two amino acid deletion, which would cause an even more radical change in the Bridge Helix by creating a local $180^{\circ}$ twist of the $\alpha$-helical structure (Figure $2 \mathrm{C}$ ). This class of mutants combines strictly confined effects (removal of two adjacent side chains) with complex long range effects (realignment of remaining side chains on either side of the deletion to new positions and localized underwinding of the helical structure), which may possibly be coupled with the propagation of stress forces to the $\mathrm{N}$ - and $\mathrm{C}$-terminal anchoring points of the Bridge Helix. The radical nature of such twisting mutations serves as an ideal tool to gain a deeper insight into structural constraints acting on the entire Bridge Helix. A series of two-amino acid deletions was prepared (Figure 4A). The results show that the $\mathrm{BH}-\mathrm{H}_{\mathrm{C}}$ region is indeed remarkably resistant to such major conformational changes (Figure 4B). Although X-ray structures provide apparently persuasive arguments for certain residues of the Bridge Helix domain assisting in catalytic functions (for example, residues orthologous to T821 contacting the 3' end of the nascent transcript and/or the incoming rNTP [22]), the deletion phenotypes described here prove that such 
(a)

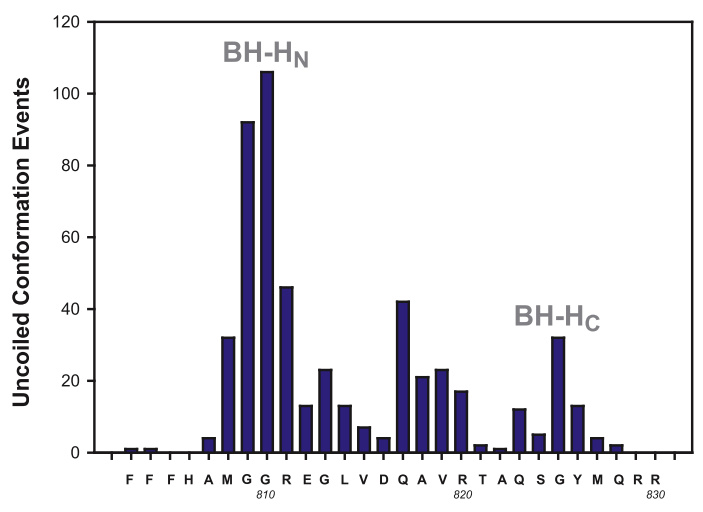

(b)

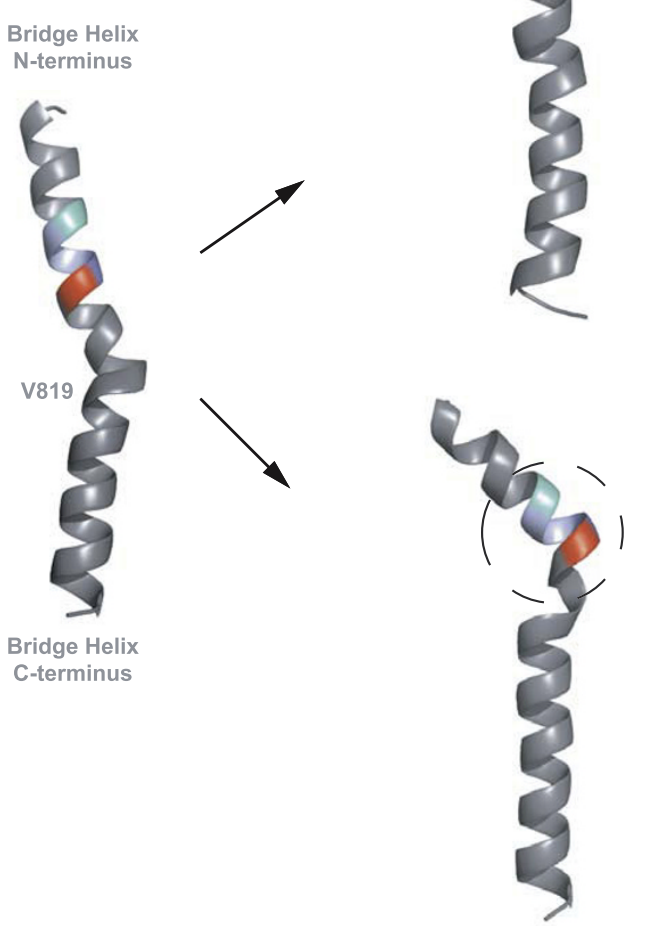

(c)
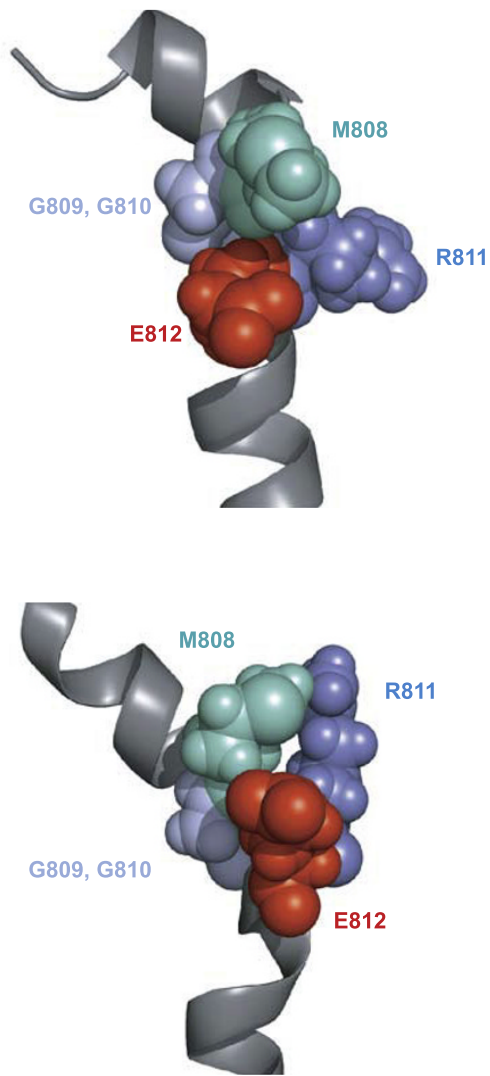

Figure 3 Structural basis of $\mathbf{B H}-\mathbf{H}_{\mathbf{N}}$ kinking. (A) Overview of local unfolding events incurred by a $\mathbf{M}$. jannaschii Bridge Helix model during 27 independent 200 picosecond MD simulations. The number of 5 picosecond windows during which a specific part of the Bridge Helix adopts a 'coil' conformation are plotted against the Bridge Helix sequence shown on the horizontal axis. A major area of $\alpha$-helical instability, BH-H $H_{N}$ (centered on $\mathrm{mjA}^{\prime} \mathrm{G} 810$ ), is evident from this semi-quantitative analysis. Additional unstable regions include $\mathrm{BH}-\mathrm{H}_{\mathrm{C}}$ (centered on $\left.\mathrm{mjA}^{\prime} \mathrm{G} 825\right)$ and a 'labile region' (spanning mjA'Q817 to R820; see Discussion for more details). (B) Examples of kinked BH-H N $_{\mathrm{N}}$ conformations arising from MD simulations. The Bridge Helix on the left represents the starting conformation as modelled on the yeast RNAPII structure PDB \#2E2H. The minor bulge near the center of the Bridge Helix corresponds to the V819 position. During unrestrained simulation, different types of kinked BH-H structures (circled) involving mjA' G809/G810 (pale blue) and M808 (pale green), R811 (blue) and E812 (red) side-chain interaction emerge stochastically. (C) Structural details of $\mathrm{BH}-\mathrm{H}_{\mathrm{N}}$ kinking models. The relevant residues are shown in space-filling mode to illustrate spatial relationships. Two glycine residues, mjA' G809 and G810 (pale blue) form a highly flexible hinge that allows M808 (pale green) to interact extensively via van der Waals interactions with the side-chains of R811 (blue) and E812 (red). In some cases these interactions create a stretched $3_{10}$ helix immediately C-terminal to R811 and E812. 
(a)

$$
\begin{aligned}
& \text { mjA' wildtype } 800 \text { PTEFFFHAMGGREGLVDQAVRTAQSGYMQRRLINAL } 836 \\
& \text { mjA' } \triangle \text { T801-E802 P--FFFHAMGGREGLVDQAVRTAQSGYMORRLINAI } \\
& \text { PT-OFHAMGGREGLVDQAVRTAOSGYMORRITNAL } \\
& \text { miA' } \triangle F 803-F 804 \square \quad \text { PTE-FFHAMGGREGLVDQAVRTAQSGYMQRRLINAL } \\
& \text { mjA' } \triangle F 804-F 805 \quad \text { PTEF--HAMGGREGLVDQAVRTAQSGYMQRRLINAI } \\
& \text { mjA' } \triangle F 805-H 806 \text { PTEFF--AMGGREGLVDQAVRTAQSGYMQRRLINAL } \\
& \text { mjA' } \triangle \text { H806-A807 PTEFFF--MGGREGLVDQAVRTAQSGYMQRRLINAL } \\
& \text { mjA' } \triangle \text { A807-M808 PTEFFFH--GGREGLVDQAVRTAQSGYMORRLINAI } \\
& \text { MJA' } \triangle A 807-M B 08 \\
& \text { mjA' } \triangle \text { G809-G810 PTEFF } \\
& \text { mjA' } \text { G810-R811 PTEFFFHAMG--EGLVDQAVRTAQSGYMQRRLINAL } \\
& \text { mjA' } \triangle \text { R811-E812 PTEFFFHAMGG--GLVDQAVRTAQSGYMQRRLINAL } \\
& \text { mjA' } \triangle \text { E812-G813 PTEFFFHAMGGR--LVDQAVRTAQSGYMQRRLINAL } \\
& \text { MIA' } \triangle \text { G813-1814 PTEFFFHAMGGRE--VDQAVRTAOSGYMORRLINAI } \\
& \text { PTEFFFHAMGGREG--DQAVRTAQSGYMQRRLINAL } \\
& \text { mjA' } \triangle \text { V815-D816 PTEFFFHAMGGREGL--QAVRTAOSGYMQRRLINAI } \\
& \text { mjA' } \triangle \text { D816-Q817 PTEFFFHAMGGREGLV--AVRTAQSGYMQRRLINAL } \\
& \text { mjA' } \triangle \text { Q817-A818 PTEFFFHAMGGREGLVD--VRTAQSGYMQRRLINAI } \\
& \text { mjA' } \triangle A 818 \text {-V819 PTEFFFHAMGGREGLVDQ--RTAQSGYMQRRLINAI } \\
& \text { MIA' } \triangle \text { V819-R820 PTEFFFHAMGGREGLVDQA--TAOSGYMQRRI INAI } \\
& \text { PTIEFFEHAMGGREGLVDQAV--AOSGYMQRRITNAL } \\
& \text { 'MA' } \triangle \text { T821-A822 PTEFFFHAMGGREGLVDQAVR--QSGYMQRRLINAI } \\
& \text { mjA' } \triangle \text { A822-Q823 PTEFFFHAMGGREGLVDQAVRT--SGYMQRRLINAL } \\
& \text { mjA' } \triangle \text { Q823-S824 PTEFFFHAMGGREGLVDQAVRTA--GYMQRRLINAI } \\
& \text { miA' } \triangle \text { S824-G825 PTEFFFHAMGGREGLVDQAVRTAQ--YMQRRLINAI } \\
& \text { 'MA' } \triangle \text { G825-Y826 PTEFFFHAMGGREGLVDQAVRTAQS--MORRI INAI } \\
& \text { PTEFEFHAMGGREGLVDQAVRTAQSG--QRRLINAL } \\
& \text { 'mjA' } \triangle \text { M827-Q828 PTEFFFHAMGGREGLVDQAVRTAQSGY--RRLINAL } \\
& \text { mjA' } \triangle \text { Q828-R829 PTEFFFHAMGGREGLVDQAVRTAQSGYM--RLINAL } \\
& m \mathrm{~A}^{\prime} \triangle \mathrm{R} 829-\mathrm{R} 830
\end{aligned}
$$

(b)

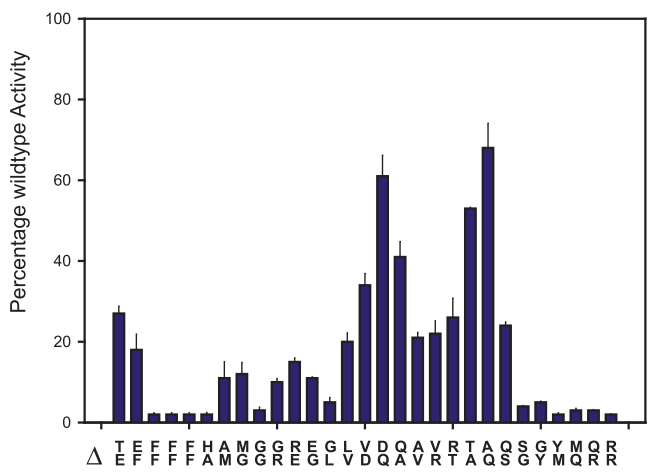

(c)

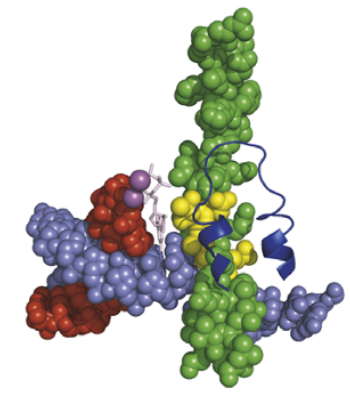

Figure 4 Creation of locally twisted Bridge Helix structures. (A) Sequences of the deletion constructs. A two-amino acid deletion window is moved systematically in a single residue step through the entire Bridge Helix primary sequence. The deletions remove two adjacent residues, but, more importantly, join the sequences bordering the mutation with $180^{\circ}$ twist because of the removal of half an $\alpha$-helical turn. (B) Activity of Bridge Helix mutants containing two-amino acid deletions shown relative to wildtype activity (100\%). The amino acid pairs deleted from the primary sequence are shown vertically along the horizontal axis. Two distinct peaks of relative insensitivity to the deletions centered on $\mathrm{mjA}^{\prime} \triangle \mathrm{D} 816 / \mathrm{Q} 817$ and mjA' $\triangle \mathrm{A} 822 / \mathrm{Q} 823$ are discernible. All assays were performed in at least quadruplicate, with error bars showing standard deviation from the average value. $(\mathbf{C})$ Position of the deletion-insensitive region of the Bridge Helix relative to other elements of the catalytic site. The Bridge Helix and other structures are shown using the same colorscheme as used in Figure 1A (yeast RNAPII elongation complex; [PDB \#2E2H]). Residues orthologous to residues displaying the highest activity (>50\%) levels in the two-amino acid scan (mjA' D816, Q817, T821, A822 and Q823) are highlighted in yellow.

contributions are either redundant or non-existent in our archaeal system, which is highly conserved in this region. The results furthermore illustrate that, although $\mathrm{BH}-\mathrm{H}_{\mathrm{C}}$ is located much closer than $\mathrm{BH}-\mathrm{H}_{\mathrm{N}}$ to the substrates involved in the NAC, there are surprisingly few topological restraints. In marked contrast, none of the two-amino acid deletions support significant activity in the N-terminal part of the Bridge Helix (Figure 4B), proving that conformational changes are much more restricted in that region (which is consistent with the higher degree of evolutionary identity in the N-terminal half of the Bridge Helix compared to the C-terminal half; Figure 1). The results from the two amino acid deletion scan thus provide a first indication that the local conformational requirements for $\mathrm{BH}-\mathrm{H}_{\mathrm{N}}$ and $\mathrm{BH}-$ $\mathrm{H}_{\mathrm{C}}$ differ quite radically.

The substitutions $m j \mathrm{~A}^{\prime} \mathrm{M} 808-\mathrm{P}$ and S824-P provide the strongest pieces of evidence for the existence and functional significance of $\mathrm{BH}-\mathrm{H}_{\mathrm{N}}$ and $\mathrm{BH}-\mathrm{H}_{\mathrm{C}}$, respectively (Figure 2B[20]). Work in other systems has shown that the physicochemical properties of residues adjacent to prolines contribute considerably to the prevalence of cis/trans peptide bond isomers and therefore strongly influence kink geometry [39-42]. Additional proline substitutions of the positions immediately $\mathrm{N}$ - and C-terminal to either M808 or S824 (that is, $m j \mathrm{~A}^{\prime} \mathrm{A} 807-\mathrm{P} /$ M808-P; M808-P/G809-P and Q823-P/S824-P; S824-P/ G825) revealed examples of further intriguing differences between $\mathrm{BH}-\mathrm{H}_{\mathrm{N}}$ and $\mathrm{BH}-\mathrm{H}_{\mathrm{C}}$ : the double-proline substitution in $\mathrm{BH}-\mathrm{H}_{\mathrm{N}}$ abolished the superactivity caused by M808-P, whereas the presence of an additional proline residue $\mathrm{N}$-terminal to $\mathrm{S} 824$ continued to support the superactivity of S824-P (Figure 5B). The activity of the Q823-P/S824-P double mutant proves explicitly that the presence of two proline residues in this particular location of the Bridge Helix is not only compatible with catalytic function, but also compatible with superactivity. Prolyl-proline preferentially adopt an elongated polyproline II structure $(87 \%)$, or less frequently (13\%) a $\beta$-turn (Additional file 4A; $[43,44]$ ). Either of the structures would cause a substantial local increase in the flexibility of $\mathrm{BH}-\mathrm{H}_{\mathrm{C}}$. 
In order to investigate this unexpected tolerance to the presence of two adjacent prolines in positions 823 and 824 in more detail, a complete substitution series of the residues around position $m j \mathrm{~A}^{\prime}$ S824-P was prepared, generating an assortment of systematic double-mutants (Figure 5A; mjA' Q823-X/S824-P and S824-P/G825-X; with $\mathrm{X}$ denoting 19 different variants). All substitutions $\mathrm{N}$-terminal to S824-P (i.e. mjA' Q823-X/S824-P) displayed an almost completely invariant degree of extensive superactivity that was indistinguishable from the original S824-P mutant (Figure 5B). This result is remarkable because previous mutagenesis of Q823 revealed a broad spectrum of activity, ranging from substantial loss of function (Q823-C or I) to superactivity (Q823-D or E; [20]). It is evident that in the doublemutants the chemical nature of the side-chain residue in position 823 exerts no further functional influence, presumably because of the major distortion already caused by the proline substitution in position 824 . Once such a gross structural alteration has occurred in S824-P, any additional changes in the adjacent $\mathrm{N}$-terminal residue become structurally irrelevant.

Because Q823-P/S824-P displayed no loss of superactivity, the residue immediately $\mathrm{N}$-terminal to the doubleproline substitution was also permutated, resulting in variants containing three adjacent substitutions in the $\mathrm{BH}-\mathrm{H}_{\mathrm{C}}$ hinge region (Figure $5 \mathrm{~A}$; $m j \mathrm{~A}^{\prime} \mathrm{A} 822-\mathrm{X} / \mathrm{Q} 823-\mathrm{P} /$ S824-P). The majority of these substitutions, including the triple proline mutant A822-P/Q823-P/S824-P (Figure $5 \mathrm{C}$; Additional file 4B), still displayed clearly detectable superactivity, albeit at a slightly reduced level. The tolerance of the $\mathrm{BH}-\mathrm{H}_{\mathrm{C}}$ hinge to radical mutagenesis, as previously observed in the two-amino acid deletion screen, is therefore also reflected in the unexpectedly high tolerance to multiple proline substitutions in that region. This geometric freedom is, however, also spatially limited: substitutions in positions C-terminal to S824-P (Figure $5 \mathrm{~A})$ were mostly inactive, indicating that despite the structural flexibility of the positions N-terminal to S824$\mathrm{P}$, the C-terminal positions are functionally constrained (Figure 5D; a similar trend is also apparent in the two amino acid deletion scan data [see Figure 4B]).

\section{$\mathrm{BH}-\mathrm{H}_{\mathrm{N}}$ and $\mathrm{BH}-\mathrm{H}_{\mathrm{C}}$ control activity of the catalytic site differently}

Taken together, the extensive body of mutagenesis data leads to the conclusion that the $\mathrm{BH}-\mathrm{H}_{\mathrm{N}}$ and $\mathrm{BH}-\mathrm{H}_{\mathrm{C}}$ structures operate within intramolecular environments that vary widely in their conformational constraints. The chemistry of the NAC is typically catalyzed by two magnesium ions, immobilized non-covalently within the active site (via the 'metal A' and 'metal B' motifs; for example, $[45,46])$. A complete substitution of $\mathrm{Mg}^{2+}$ by $\mathrm{Mn}^{2+}$ reduces the activity of archaeal RNAP to approximately 40\% (data not shown); the reduced activity is probably caused by the slightly larger $\mathrm{Mn}^{2+}$ ions causing suboptimal spacing [47], and $\mathrm{Mn}^{2+}$-catalyzed reactions being more promiscuous in their requirements for alignment of the reactive groups [48]. Intriguingly, assays of superactive $\mathrm{BH}-\mathrm{H}_{\mathrm{N}}$ and $\mathrm{BH}-\mathrm{H}_{\mathrm{C}}$ mutants in the presence of $\mathrm{Mn}^{2+}$ demonstrated that they fell into distinct categories. mjA' M808-D, M808-E and M808-P continued to display superactivity, whereas the activities of S824-P or Q823-P/S824-P only reached wildtype levels under these conditions (Figure 6). We can therefore conclude that the superactive substitutions in the $\mathrm{BH}-\mathrm{H}_{\mathrm{N}}$ and $\mathrm{BH}-\mathrm{H}_{\mathrm{C}}$ regions have different consequences because conformational changes in different parts of the Bridge Helix affect separate stages of the NAC. The reduced catalytic activity caused by the presence of $\mathrm{Mn}^{2}$ + ions in the active site becomes rate-limiting in Q823D/E or S824-P, whereas M808-P overcomes this limitation to a large extent by stimulating transcription through an independent pathway, most likely involving the $\beta$-D II and Link domains.

\section{Discussion}

\section{An expanded conformational repertoire of the Bridge Helix domain}

The results presented here reveal several new surprising insights, including compelling evidence for the existence of a molecular hinge region in the $\mathrm{N}$-terminal portion of the Bridge Helix and evidence for an unexpectedly large degree of tolerance to radical structural changes in the C-terminal part of this domain. It is apparent that the Bridge Helix domain displays a much greater conformational freedom than anticipated from currently available X-ray structures of RNAPs. Few, if any, of the residues of the Bridge Helix appear to make any specific contribution to catalysis other than through defining the nanomechanical properties intrinsic to the $\alpha$-helical structure. The implications for mechanistic models aimed at describing the NAC are manifold, ranging from a re-evaluation of the structural basis of the RNAP translocation mechanism, to highlighting the hitherto neglected role of highly conserved domains in the catalytic site, and to obtaining a better understanding of the evolutionary diversity of Bridge Helices in different organisms.

Currently, we have only a limited understanding of the forces acting on the Bridge Helix that could drive localized conformational changes. Attempts to model the full NAC using molecular dynamics studies are severely limited by the large size of multi-subunit RNAPs and the immense computational effort that would be required to simulate the molecular events expected to last from $10 \mathrm{~s}$ to $100 \mathrm{~s}$ of milliseconds for the extension of a nascent transcript by a single nucleotide (approximately $30 \mathrm{~ms}$ / 
(a)

$$
\begin{aligned}
& \text { S. cerevisiae } \\
& \text { M. jannaschii wildtype } \\
& \text { S824-P } \\
& \text { Q823-X/S824-P } \\
& \text { A822-X/Q823-P/S824-P } \\
& \text { S824-P/G825-X } \\
& \text { S824-P/G825-P/Y826-X }
\end{aligned}
$$

$\underset{1}{\mathrm{BH}_{\mathrm{C}}}$

10 PQEFFFHAMGGREGLIDTAVKTAETGIIQRRLVKAL 845

800 PTEFFFHAMGGREGLVDQAVRTAQSGYMQRRLINAL 83

PTEFFFHAMGGREGLVDQAVRTAQPGYMQRRLINAL

PTEFFFHAMGGREGLVDQAVRTAXPGYMQRRLINAL

PTEFFFHAMGGREGLVDQAVRTXPPGYMQRRLINAL

PTEFFFHAMGGREGLVDQAVRTAOPXYMORRL INAI

PTEEEEHAMGRREGUDQAVRTAQP PXMQRRLINAT

(b)
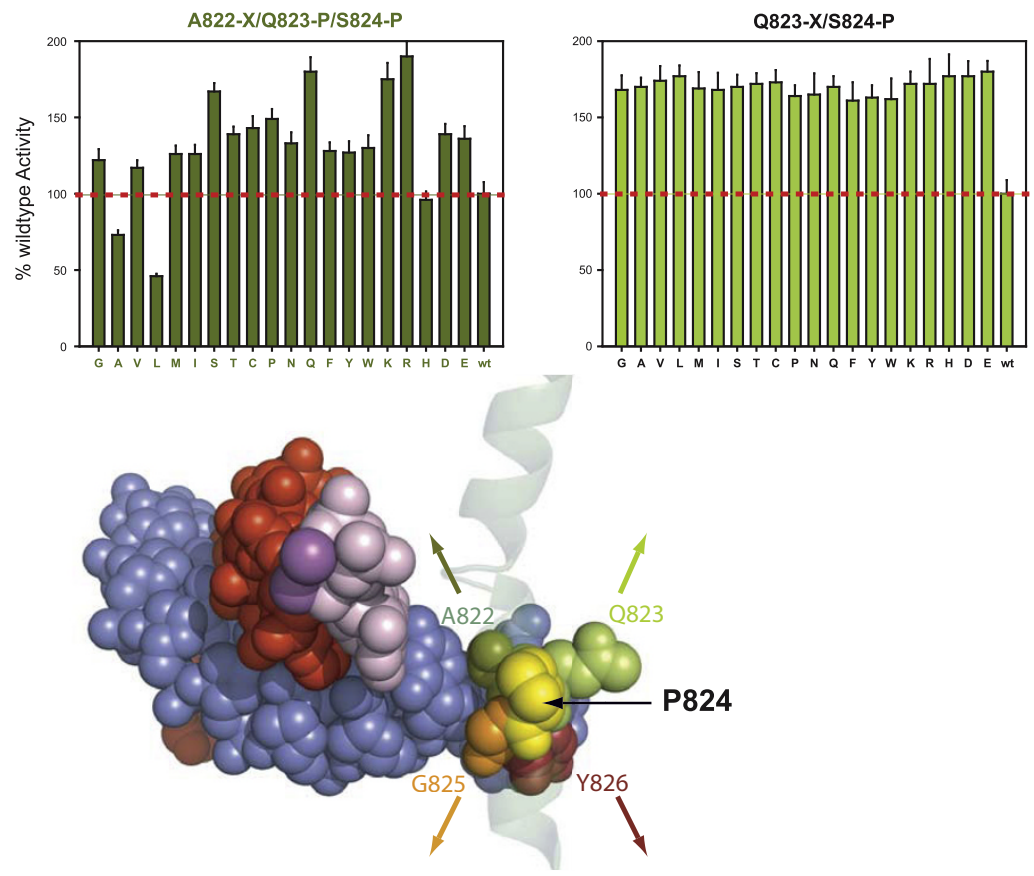

S824-P/G825-X
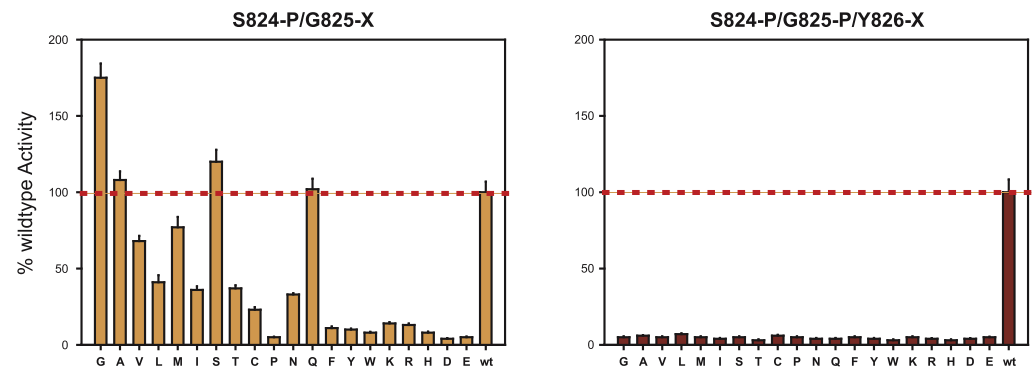

Figure 5 Functional dissection of the $\mathbf{B H}-\mathrm{H}_{\mathbf{C}}$ region. (A) Position of mutagenized residues. The residues that differ from the archaeal wildtype sequence in the various mutagenesis constructs are shown in red. Wildtype sequences of the M. jannaschii and S. cerevisiae Bridge Helix sequences are shown for comparison. The position of $\mathrm{BH}-\mathrm{H}_{\mathrm{C}}$ is marked with an arrow. (B) The center of the panel provides an overview depicting the orthologous residues in space-filling mode within the mutagenized part of the Bridge Helix modelled on the yeast RNAPII structure (PDB \#2E2H); yeast residues E823 and T824 were replaced in silico with Q and P, respectively, to reveal the approximate location of these amino acids relative to the DNA-RNA hybrid and catalytic site). Adjacent parts of the Bridge Helix domain are shown as a transparent ribbon. All other colors are coded as in Figure 1A (template DNA is pale blue, the nascent transcript is red, the NTP in the insertion site is pink and catalytic metal ions are shown as magenta spheres). All mutants shown here contain the mjA' S824-P substitution (yellow). The results of promoter-independent activity assays are plotted relative to wildtype activity (indicated with a red dashed line). Two of the bar charts show the effect of introducing systematic substitutions in positions located immediately N- or C-terminal to S824-P (mjA' Q823 [lime green; upper right]; mjA' G825 [orange; lower left]). The other two bar charts show the functional consequences of introducing additional systematic substitutions in positions located immediately N-terminal to the double proline substitution Q823-P/S824-P (mjA' A822 [olive green; upper left]), or immediately C-terminal to the double proline substitution S824-P/G825 (mjA' Y826 [brown; lower left]). The colors of the histogram bars match the colors of the substituted residues in the structural model. All assays were performed in at least quadruplicate, with error bars showing standard deviation from the average value. 


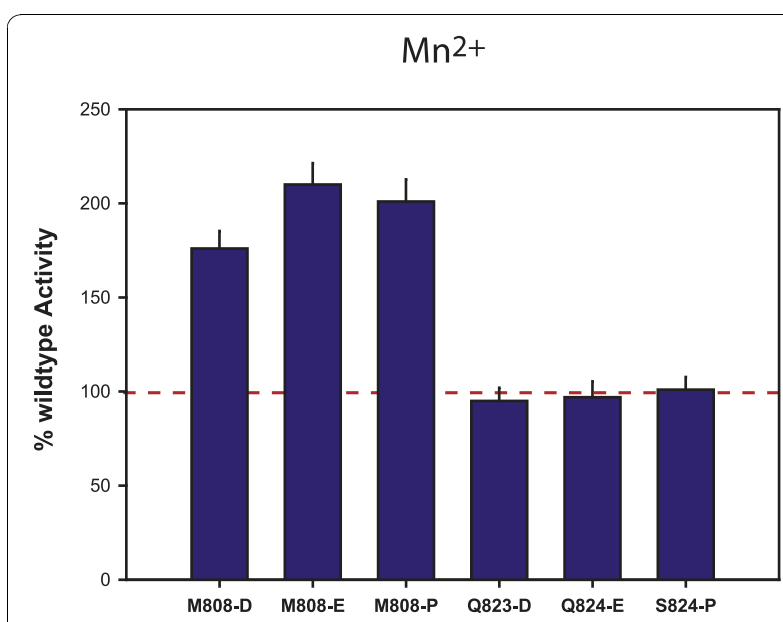

Figure 6 Differential effect of $\mathrm{Mn}^{2+}$ on superactivity in the $\mathrm{BH}$ $\mathrm{H}_{\mathrm{N}}$ and $\mathrm{BH}-\mathrm{H}_{\mathrm{C}}$ Region. The activities of the most superactive substitutions in $\mathrm{BH}-\mathrm{H}_{\mathrm{N}}$ and $\mathrm{BH}-\mathrm{H}_{\mathrm{C}}$ are compared in $\mathrm{Mn}^{2+}$ substituted reactions relative to the wildtype enzyme. The $\mathrm{BH}-\mathrm{H}_{\mathrm{N}}$ substitutions ( $m j A^{\prime}$ M808-D, -E and -P) continue to display a high degree of superactivity, whereas $B H-H_{C}$ substitutions ( $m j A^{\prime}$ Q823-D, -E and S824-P) only transcribe at rates comparable to wildtype RNAP.

rNTP incorporation under optimal in vivo conditions; for example, [49]). The study of the intrinsic structural properties of individual domains by fully atomistic computer simulations reveal, however, interesting nanomechanical properties that have functional implications for the RNAP translocation mechanism [50-52]. The Bridge Helix domain contains intrinsically unstable $\alpha$-helical regions that undergo spontaneous kinking motions, even in the absence of externally applied forces (Figure 3A). At least two of these unstable regions correspond precisely to the biochemically-mapped $\mathrm{BH}-\mathrm{H}_{\mathrm{N}}$ and $\mathrm{BH}-\mathrm{H}_{\mathrm{C}}$ regions. Strategically-placed glycine residues, such as mjA' G809, G810 (for BH- $\mathrm{H}_{\mathrm{N}}$ ) and G825 (for BH- $\mathrm{H}_{\mathrm{C}}$ ) provide the structural basis for forming these molecular hinges, with surrounding residues determining additional kinking parameters, such as the likelihood of kinking and/or the half-life of the kink after its isomerization (Figure 2A; Heindl et al., unpublished observations). Interestingly, the simulations also highlight a potential third structurally labile region near the center of the Bridge Helix domain, spanning residues mjA' Q817 to R820. The relative sensitivity of this sequence to proline substitutions (Figure 2B) suggests, however, that this area of instability behaves functionally differently to $\mathrm{BH}-\mathrm{H}_{\mathrm{N}}$ and $\mathrm{BH}-\mathrm{H}_{\mathrm{C}}$. It is possible that structural fluctuations in the central part of the Bridge Helix play a more dynamic role in supporting short-lived conformational changes that can either compensate for major structural rearrangements due to $\mathrm{BH}-\mathrm{H}_{\mathrm{N}}$ and $\mathrm{BH}-\mathrm{H}_{\mathrm{C}}$ kinking, or act as a store of 'fast' motions to lubricate the kinking of the hinge regions kinetically [34]. Although such hypotheses are currently beyond experimental proof, it is interesting to note that several, structurally unexplained superactivity mutants map to this central sequence (for example, mjA' D816-N; Q817-S/T/C/K and V819-K;Figure 2A [20]), and that this region is also exceptionally tolerant to radical twisting of the $\alpha$-helical axis induced by deletions of two-amino acid segments (Figure 4B). Furthermore, data from anisotropic network mode analysis suggests that rigid body movements of the clamp domain may exert forces onto the center of the Bridge Helix via the Switch domains, potentially linking transcription to a ratchet-like translocation mechanism (Additional file 5; [53]). The Bridge Helix thus appears to have evolved specific nanomechanical properties that result in the controlled and highly localized isomerization of its conformation in response to allosteric alterations in the surrounding protein domains and nucleic acid substrates.

The Bridge Helix N-terminus is tightly surrounded by other domains, such as the $\beta$-D loop II [54], the experimentally uncharacterized Link domain $[25,55]$, and the F-Loop [26]. As evident from the exceptionally high degree of sequence conservation (Additional files 1A, $2 \mathrm{~A}$ and $3 \mathrm{~A}$ ), each of these domains is likely to play key roles in the NAC. The $\beta$-D II domain is a loop-like structure that interacts extensively in a side-way interaction with the central part of the Bridge Helix, while simultaneously maintaining direct physical contact with the most recently incorporated nucleotide (i-1 position). The interaction between the $\beta$-D II domain and nascent transcript also creates an extended binding pocket for the rNTP (additional file 1B, C). Similarly, the highly conserved Link domain is strategically placed to interact with the Bridge Helix N-terminus, $\beta$-D II domain, nascent transcript (i-1 and $\mathrm{i}-2$ positions) and the incoming rNTP (Additional file 2B). Finally, an N-terminal extension of the Bridge Helix, the F-Loop, forms an extensive cap-like structure that contacts the Link domain and the tip of the Trigger Loop (Additional file 3B; [26]). The differential response of superactive substitutions in $\mathrm{BH}$ $\mathrm{H}_{\mathrm{N}}$ and $\mathrm{BH}-\mathrm{H}_{\mathrm{C}}$ to the presence of $\mathrm{Mn}^{2+}$ in the catalytic site supports the view that conformational changes in these regions cause a distinct effect in the catalytic site of RNAP. While the C-terminal Bridge Helix operates predominantly by influencing Trigger Loop conformation, kinking of the $\mathrm{N}$-terminus via $\mathrm{BH}-\mathrm{H}_{\mathrm{N}}$ most likely alters the positions and/or conformations of the $\beta$-D II and Link domains, which are in direct physical contact with the nucleotide and nucleic acid substrates.

\section{In vivo occurrence of proline-substituted Bridge Helix hinges}

The existence and biological relevance of the experimentally determined hinge positions that tolerate proline substitutions is independently confirmed by a small 
number of naturally occurring Bridge Helix variants. Considering the large number of genomes sequenced thus far (currently including approximately 1,200 microbial and approximately 800 eukaryotic genomes; http:// www.ncbi.nlm.nih.gov/sites/genome/), it can already be stated with confidence that naturally occurring proline substitutions are an exceedingly rare phenomenon. The three bacterial species that contain naturally occurring proline-substitutions in $\mathrm{BH}-\mathrm{H}_{\mathrm{N}}$ represent two diverse bacterial phyla (Figure 7), and each of these phyla contains other closely related species with sequenced genomes, which do not contain any prolines in their Bridge Helices. Proline substitutions in the $\mathrm{BH}-\mathrm{H}_{\mathrm{N}}$ region therefore appear to evolve spontaneously and independently in different bacterial lineages and subsequently remain restricted to individual species or strains. In at least some cases this evolutionary adaptation may be associated with significant simplifications of the transcriptional machinery, such as loss of the $\omega$-RNAP subunit and absence of transcription-coupled repair $(O$. tsutsugamushi; $[28,29])$. These proline-containing Bridge Helix variants do not only provide strong and independent in vivo confirmation of the results identified in the high-throughput mutagenesis screen (Figure 2B), but prove that the results obtained in an archaeal model system also apply to the bacterial and eukaryotic domains; Bridge Helix kinking via two structurally independent molecular hinges is a universal mechanism operating across the entire evolutionary spectrum.

\section{Conclusions}

The application of a high-throughput in vitro mutagenesis approach to the $\mathrm{N}$-terminal portion of the $M$. jannaschii bridge helix domain has revealed a range of new insights that could not be anticipated from previously available structural and genetic data. The data sets (Figures $2 \mathrm{~A}, 4 \mathrm{~B}$ and $5 \mathrm{~B}$ ) clearly illustrate that many of the most interesting insights were derived from substitutions that would almost certainly not have been designed using a rational, structure-led approach (for example, mjA' M808-P; $\Delta$ D816/Q817; A822-P/Q823-P/ S824-P). Furthermore, widely used methods, based predominantly on alanine-scanning mutagenesis [56], are also limited in their capacity to uncover some of the most interesting phenotypes (Figure 2A). It is therefore clear that automated high-throughput methods for generating site-directed mutants and assaying their phenotypic consequences will play an increasingly important role in exploratory investigations of protein structure/ function relationships as part of a diverse strategy aimed at obtaining new insights into complex biological systems [57].

\section{Methods}

\section{High-throughput mutagenesis}

Combinatorial permutation libraries, containing all 19 variants with codon replacements optimized for expression in $E$. coli were purchased for $m j \mathrm{~A}^{\prime} \mathrm{H} 806, \mathrm{~A} 807$, M808, G809, G810, R811, E812, G813, Q823-X/S824-P, A822-X/Q823-P/S824-P, S824-P/G825-X, and S824-P/ G825-P/Y826-X from GeneArt (Regensburg, Germany). The mutations, located within a BstBI-SbfI fragment of the codon-optimized C-terminus of the Bridge Helix [19] were transferred to a pET21a bacterial expression vector for the production of full-length, intein-free $m j \mathrm{~A}^{\prime}$ subunits. The presence of the desired mutations in the expression constructs was verified by DNA sequencing. DNA constructs containing the two amino-acid deletions across the Bridge Helix were purchased from GeneArt as synthetic gene fragments and transferred to bacterial expression plasmids as described above.

\section{RNAP Factory}

The robotic procedures for high-throughput growth of bacterial expression strains, recombinant subunit purification and automated assembly into recombinant RNAPs (the 'RNAP Factory') have been described previously $[19,20,58]$. Briefly, bacterial constructs expressing the Bridge Helix mutants were transformed into chemically competent Acella cells ( $\triangle e n d A \Delta r e c A$ derivatives of E. coli BL21 [DE3]; EdgeBio, Gaithersburg, Maryland, USA). After growth for 16 to 18 hours at $37^{\circ} \mathrm{C}$ in 24 well plates in autoinduction medium (OverNight Express, Novagen, Nottingham, UK) the bacteria were harvested and used for a centrifugation-based robotic inclusion body purification protocol. The $m j \mathrm{~A}^{\prime}$ inclusion bodies were automatically solubilised in the presence of $8 \mathrm{M}$ urea and quantitated at $562 \mathrm{~nm}$ with the bicinchoninic acid assay. Recombinant RNAPs containing the $m j \mathrm{~A}^{\prime}$ variants were assembled in a 96-well format dialysis cell using an urea-gradient from $6 \mathrm{M}$ to urea-free spanning 16 hours at room temperature [19]. Each mutant subunit was expressed, purified and assembled in vitro at least in quadruplicate to assure consistency and reproducibility. The assembled RNAPs were harvested and used immediately for robotic transcription assays (see below). The assembly efficiencies of key mutants (including, among others, mjA' M808-P; A807P/M808-P; M808-P/G809-P; S824-P; Q823-P/S824-P; S824-P/G825-P; A822-P/Q823-P/S824-P) were compared to assembly rates achieved with the wildtype $m j \mathrm{~A}^{\prime}$ subunit by assaying the reconstituted polymerases at limiting and saturating template DNA concentrations (see Additional file 19 in [20] for details and examples); no differences between wild-type and mutant enzymes 


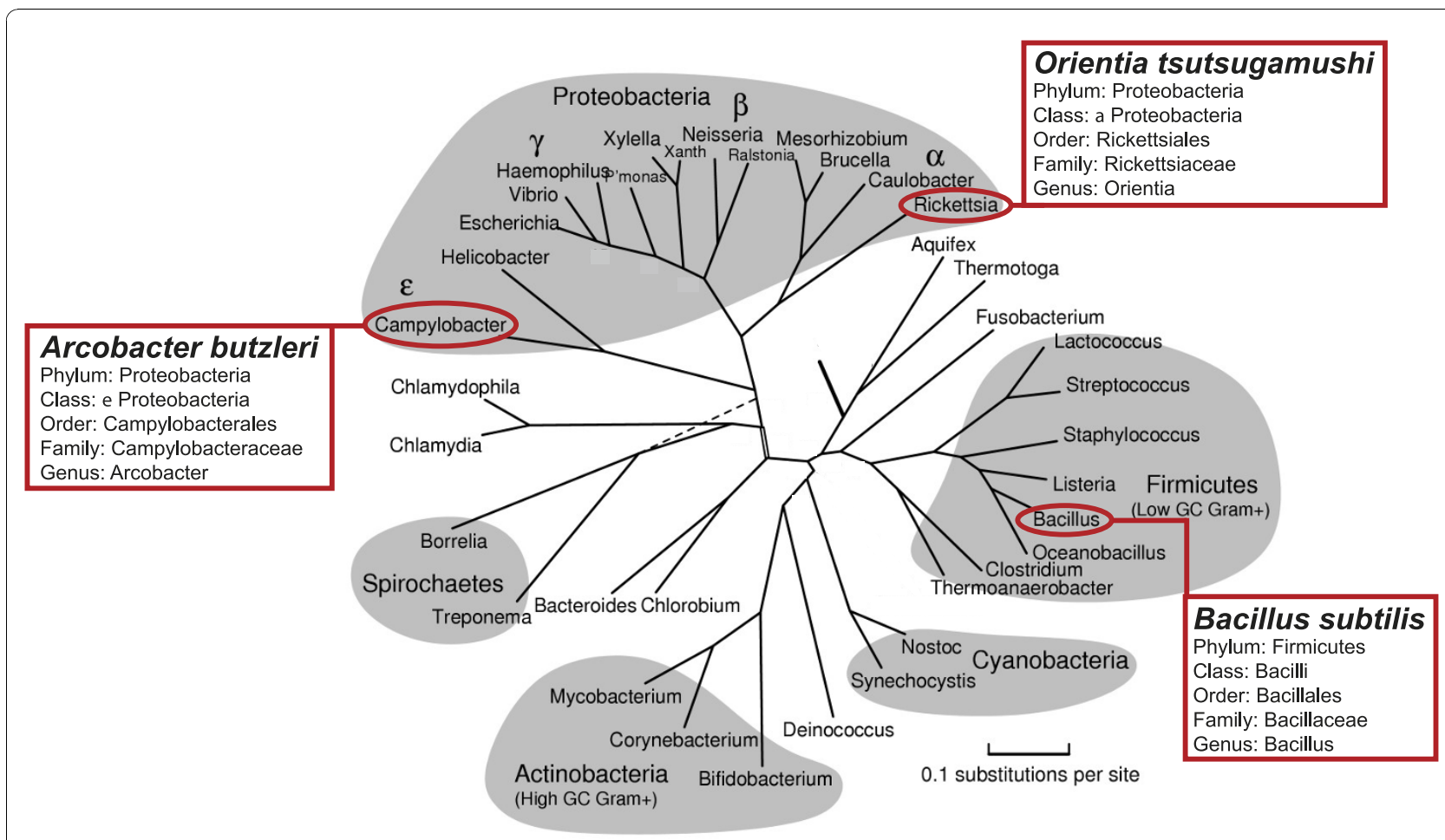

Figure 7 Evolutionary positions of bacterial species with proline-containing Bridge Helices. The evolutionary relationship between three bacterial species with proline-containing Bridge Helices is shown on a rooted phylogenetic tree calculated using maximum-likelihood methods from a concatenation of representative protein sequences [63]. The three species occupy widely divergent branches, strongly suggesting that the proline substitutions evolved independently, rather than were derived from a recently shared ancestor.

were detected. For replacement of $\mathrm{Mg}^{2+}$ ions in the catalytic site with $\mathrm{Mn}^{2+}$ (for the transcription assays shown in Figure 6), the in vitro assembly process was carried out as described above, but with dialysis- and transcription buffers containing $10 \mathrm{mM} \mathrm{Mn}_{2}$-O-acetate instead of $\mathrm{Mg}_{2}$-O-acetate.

\section{In vitro transcription assays}

The robotically implemented high-throughput trichloroacetic acid (TCA) precipitation assays, measuring the incorporation of $\left(\alpha-{ }^{32} \mathrm{P}\right)$ rUTP into TCA-insoluble products, were carried out exactly as previously described $[19,20]$. Briefly, assay mixtures were incubated for 45 minutes at $70^{\circ} \mathrm{C}$ in thin-wall PCR plates before precipitating the radiolabeled transcripts by the addition of icecold TCA solution. After incubation for 30 minutes at $1^{\circ} \mathrm{C}$, the nucleic acid precipitates were collected by vacuum filtration on a $96-\mathrm{GF} / \mathrm{F}$ glass fiber filter plate (Whatman, Maidstone, UK) and extensively washed with further aliquots of ice-cold TCA solution. After drying the filters, scintillant (MicroScint-O; PerkinElmer, Cambridge, UK) was added and the amount of incorporated $\left(\alpha-{ }^{32} \mathrm{P}\right)$ rUTP quantified with a microplate counter (TopCount NXT, Packard, Cambridge, UK).
High-throughput molecular dynamics simulation

Molecular dynamics (MD) simulations were performed using GROMACS [59]. In preparation for MD simulations, the archaeal Bridge Helix was modelled on the $S$. cerevisiae 'active elongation' RNAPII structure (PDB $\# 2 \mathrm{E} 2 \mathrm{H}$ ) using the SwissModel server in automated mode [60]. The simulation production runs were executed in a fully solvated atomistic production mode without restraints. The energies of the modelled structures were initially minimized in vacuum using GROMACS with an AMBER force field (http://ambermd.org/ ) on a CPU cluster of the National Grid Service (NGS). During pre-processing the system was warmed to $200 \mathrm{~K}$ under the control of a Berendsen thermostat with a coupling constant of 1.0 ps. All structures were energyminimized in pre-equilibrated simulation boxes filled with TP3 water, and sodium and chloride ions were added to a final concentration of approximately 150 $\mathrm{mM}$. For production runs the temperature was increased to $300 \mathrm{~K}\left(27^{\circ} \mathrm{C}\right)$. The equations of motion were integrated using a step-size of two femtoseconds. The trajectories generated by 27 independent 200 picoseconds simulation runs, were analyzed using STRIDE [61], as implemented in VMD [62]. The frequencies of 
particular residues adopting a 'coil' conformation during 5 ps analysis windows were plotted relative to the Bridge Helix sequence.

\section{Additional material}

Additional file 1: Evolutionary conservation and structure of the $\beta$ D II domain. (A) Alignment of $\beta-D$ II domain sequences from bacteria ( $E$. coli K12, T. thermophilus) and eukaryotes (S. cerevisiae and H. sapiens) against the archaeon M. jannaschii. Residues identical to the archaeal sequence are shown in red. The numbers flanking the sequences represent the location of the sequences within the open reading frame of the complete subunit. One of the residues in close contact with the nascent transcripts is boxed and identified with an arrow. (B) Arrangement of the $\beta-D$ II domain relative to the RNAP active site. Most structures are shown in space-filling mode to emphasize spatial connections. The Bridge Helix is shown in green (with the $\mathrm{BH}-\mathrm{H}_{\mathrm{N}}$ region (corresponding to $\mathrm{mjA}^{\prime} \mathrm{M} 808-\mathrm{E} 812$ ) highlighted in yellow), the $\beta$-D II domain in turquoise, the template DNA is pale blue, the RNA is red, the NTP in the insertion site shown as a pink stick model and catalytic metal ions as magenta spheres. The rNTP binding pocket is indicated with a white-dashed oval. (C) Close-up view of the $\beta$-D II domain. Note the extensive contacts between the $\beta-\mathrm{D} \|$ domain with the rNTP and the i-1 position of the nascent transcript. Two potential $\beta$-D II/Bridge Helix contacts are mediated via residues orthologous to mjA' R811 and L814. ((PDB \#2E2H); visualized with PyMOL).

\section{Additional file 2: Evolutionary conservation and structure of the}

Link domain. (A) Alignment of Link domain sequences from bacteria (E. coli K12, T. thermophilus) and eukaryotes (S. cerevisiae and H. sapiens) against the archaeon $M$. jannaschii. Residues identical to the archaeal sequence are shown in red. The numbers flanking the sequences represent the location of the sequences within the open reading frame of the complete subunit. Residues in close contact with the rNTP or nascent transcripts are indicated by boxes and arrows. (B) Arrangement of the Link domain relative to the RNAP active site. Most structures are shown in space-filling mode to emphasize spatial connections. The Bridge Helix is shown in green (with the $\mathrm{BH}-\mathrm{H}_{\mathrm{N}}$ region (corresponding to mjA' M808-E812) highlighted in yellow), the Link domain in light purple, the template DNA is pale blue, the RNA is red, the NTP in the insertion site shown as a pink stick model and catalytic metal ions as magenta spheres. The rNTP binding pocket is indicated with a white-dashed oval.

Additional file 3: Evolutionary conservation and structure of the Link domain. (A) Alignment of F-Loop domain sequences from bacteria (E. coli K12, T. thermophilus) and eukaryotes (S. cerevisiae and H. sapiens) against the archaeon $M$. jannaschii. Residues identical to the archaeal sequence are shown in red. The numbers flanking the sequences represent the location of the sequences within the open reading frame of the complete subunit. (B) Arrangement of the F-Loop domain relative to the RNAP active site. Most structures are shown in space-filling mode to emphasize spatial connections. The Bridge Helix is shown in green (with the $\mathrm{BH}-\mathrm{H}_{\mathrm{N}}$ region (corresponding to $\mathrm{mjA}^{\prime} \mathrm{M} 808-\mathrm{E} 812$ ) highlighted in yellow), the F-Loop domain in lime, the template DNA is pale blue, the RNA is red, the NTP in the insertion site shown as a pink stick model and catalytic metal ions as magenta spheres.

\section{Additional file 4: Analysis of proline conformational space in} proteins. (A) Conformation of peptide backbones containing a single ( $X$ $P-X ; X$ is any other non-proline residue; left panel), two (X-P-P-X; central panel), or three subsequent proline residues (X-P-P-P-X) in protein structures displayed as Ramachandran plots (data generated using the web-based server described in [64]). The relative frequency of occurrence of particular $\phi / \omega$ angle is encoded by the brightness of the square at the intersection of the coordinates. The $\phi / \omega$ angle combination compatible with standard $\alpha$-helical conformation is indicated with a dashed purple oval (left and central panels). Single proline residues conform to $\alpha$-helical geometry when present at the extreme $\mathrm{N}$ - and $\mathrm{C}$-termini of the $\alpha$-helix, thus accounting for the occurrence of single prolines in the $\alpha$-helical part of the plot in the left panel. For two or three adjacent proline residues, the only conformational space is in the top left quadrant of the plot, corresponding to polyproline-specific conformations. (B) Model of the extended poly-proline stretch in the $\mathrm{mjA}^{\prime}$ A822-P/Q823-P/S824-P triple proline substitution mutant. The triple substitution mutant displays approximately $150 \%$ of activity in comparison to the wildtype enzyme (Figure 4B). The three proline substitutions are shown as yellow stick models and T821 is shown in red as a reference point pointing towards the catalytic site. The structure shown here was constructed using the M. jannaschii Bridge Helix sequence and conforms to the typical $\phi / \omega$ angle combinations observed in $\alpha$-helices and in polyproline structures. The structure is not necessarily an accurate model, but serves to demonstrate the increased local flexibility due to the presence of three subsequent proline residues. The model was created with Abalone http://www.biomolecular-modeling.com/ Abalone/index.html.

Additional file 5: Gaussian Network Model Analysis. The yeast RNAP elongation complex (PDB \#2E2H) was subjected to Gaussian Network Model simulation (ignm.ccbb.pitt.edu; $6 \AA$ cut-off) to assess the distribution of forces within intact RNAPs. Slow-mode motions (rank 4,6 and 8) affect particularly the center of the Bridge Helix (as indicated by green/gold color-coding), whereas the $\mathrm{N}$ - and $\mathrm{C}$-terminal region remain immobile. The slow modes identify predominantly the response of individual domains to mechanical forces exerted on them from other structures during the simulation. It should be noted that such simulations do not take the chemical nature of residues into account and would therefore not be able to detect the intrinsic kinking properties of the $\mathrm{BH}-\mathrm{H}_{\mathrm{N}}$ and the $\mathrm{BH}-\mathrm{H}_{\mathrm{C}}$ regions.

\section{Abbreviations}

$\mathrm{BH}-\mathrm{H}_{\mathrm{c}}$ : molecular hinge located within the carboxy-terminal portion of the Bridge Helix; $\mathrm{BH}-\mathrm{H}_{\mathrm{N}}$ : molecular hinge located within the amino-terminal portion of the Bridge Helix; NAC: nucleotide addition cycle; rNTP: ribonucleotide triphosphate; ps: picosecond; RNAP: RNA polymerase; TCA: trichloroacetic acid.

\section{Acknowledgements}

This work was supported by a Wellcome Trust project grant [078043/Z/05/Z] to ROJW. I would like to thank Dominic Conquest for help with the subcloning of the M808-X and G813-X expression clone libraries into expression vectors. I would also like to thank Hans Heindl for advice on the molecular dynamics simulations, Noam Weingarten for programming and Tamas Kiss and Gabor Terstyanszki for advice and provision of computing resources on the UK National Grid Service. I also appreciate the helpful comments on the manuscript received from Martin Buck, Patricia Burrows and Simone Wiesler.

Received: 20 September 2010 Accepted: 29 October 2010 Published: 29 October 2010

\section{References}

1. Brueckner F, Ortiz J, Cramer P: A movie of the RNA polymerase nucleotide addition cycle. Curr Opin Struct Biol 2009, 19:294-299.

2. Erie DA, Kennedy SR: Forks, pincers, and triggers: the tools for nucleotide incorporation and translocation in multi-subunit RNA polymerases. Curr Opin Struct Biol 2009, 19:708-714.

3. Svetlov V, Nudler E: Macromolecular micromovements: how RNA polymerase translocates. Curr Opin Struct Biol 2009, 19:701-707.

4. Kireeva M, Kashlev M, Burton ZF: Translocation by multi-subunit RNA polymerases. Biochim Biophys Acta 2010, 1799:389-401.

5. Zhang G, Campbell EA, Minakhin L, Richter C, Severinov K, Darst SA: A crystal structure of Thermus aquaticus core RNA polymerase at $3.3 \AA$ resolution. Cell 1999, 98:811-824.

6. Cramer P, Bushnell DA, Fu J, Gnatt AL, Maier-Davis B, Thompson NE, Burgess RR, Edwards AM, David PR, Kornberg RD: Architecture of RNA polymerase II and implications for the transcription mechanism. Science 2000, 288:640-649

7. Cramer P, Bushnell DA, Kornberg RD: Structural basis of transcription: RNA polymerase II at 2.8. Å resolution. Science 2001, 292:1863-1876.

8. Vassylyev DG, Sekine SI, Laptenko O, Lee J, Vassylyeva MN, Borukhov S, Yokoyama S: Crystal structure of a bacterial RNA polymerase holoenzyme at $2.6 \AA$ A resolution. Nature 2002, 417:712-719. 
9. Korkhin Y, Unligil UM, Littlefield O, Nelson PJ, Stuart DI, Sigler PB, Bell SD, Abrescia NG: Evolution of complex RNA polymerases: the complete archaeal RNA polymerase structure. PLOS Biol 2009, 5:7.

10. Gnatt AL, Cramer P, Fu J, Bushnell DA, Kornberg RD: Structural basis of transcription: an RNA polymerase II elongation complex at $3.3 \mathrm{~A}$ resolution. Science 2001, 292:1876-1882

11. Westover KD, Bushnell DA, Kornberg RD: Structural basis of transcription: separation of RNA from DNA by RNA polymerase II. Science 2004, 303:1014-1016.

12. Wang D, Bushnell DA, Westover KD, Kaplan CD, Kornberg RD: Structural basis of transcription: role of the trigger loop in substrate specificity and catalysis. Cell 2006, 127:941-954.

13. Vassylyev DG, Vassylyeva MN, Perederina A, Tahirov TH, Artsimovitch I: Structural basis for transcription elongation by bacterial RNA polymerase. Nature 2007, 448:157-162.

14. Brueckner F, Cramer P: Structural basis of transcription inhibition by alpha-amanitin and implications for RNA polymerase II translocation. Nat Struct Mol Biol 2008, 15:811-818.

15. Kaplan $C D$, Larsson KM, Kornberg RD: The RNA polymerase II trigger loop functions in substrate selection and is directly targeted by alphaamanitin. Mol Cell 2008, 30:547-556.

16. Borukhov S, Nudler E: RNA polymerase: the vehicle of transcription. Trends Microbiol 2008, 16:126-134.

17. Vassylyev DG: Elongation by RNA polymerase: a race through roadblocks. Curr Opin Struct Biol 2009, 19:691-700.

18. Werner F, Weinzierl RO: A recombinant RNA polymerase II-like enzyme capable of promoter-specific transcription. Mol Cell 2002, 10:635-646.

19. Nottebaum S, Tan L, Trzaska D, Carney HC, Weinzierl RO: The RNA polymerase factory: a robotic in vitro assembly platform for highthroughput production of recombinant protein complexes. Nucleic Acids Res 2008, 36:245-252

20. Tan L, Wiesler S, Trzaska D, Carney HC, Weinzierl RO: Bridge helix and trigger loop perturbations generate superactive RNA polymerases. J Biol 2008, 7:40.

21. Klug A: Structural biology. A marvellous machine for making messages. Science 2001, 292:1844-1846.

22. Bar-Nahum G, Epshtein V, Ruckenstein AE, Rafikov R, Mustaev A, Nudler E: A ratchet mechanism of transcription elongation and its control. Cell 2005, 120:183-193.

23. Sousa R: Machinations of a Maxwellian demon. Cell 2005, 120:155-156.

24. Kaplan CD, Kornberg RD: A bridge to transcription by RNA polymerase. $J$ Biol 2008, 7:39.

25. Weinzierl RO: Nanomechanical constraints acting on the catalytic site of cellular RNA polymerases. Biochem Soc Trans 2010, 38:428-432.

26. Miropolskaya N, Artsimovitch I, Klimasauskas S, Nikiforov V, Kulbachinskiy A: Allosteric control of catalysis by the F loop of RNA polymerase. Proc Natl Acad Sci USA 2009, 106:18942-18947.

27. Barlow DJ, Thornton JM: Helix geometry in proteins. J Mol Biol 1988, 201:601-619.

28. Cho NH, Kim HR, Lee JH, Kim SY, Kim J, Cha S, Kim SY, Darby AC, Fuxelius HH, Yin J, Kim JH, Kim J, Lee SJ, Koh YS, Jang WJ, Park KH, Andersson SG, Choi MS, Kim IS: The Orientia tsutsugamushi genome reveals massive proliferation of conjugative type IV secretion system and host-cell interaction genes. Proc Natl Acad Sci USA 2007, 104:7981-7986.

29. Nakayama K, Yamashita A, Kurokawa K, Morimoto T, Ogawa M, Fukuhara M, Urakami H, Ohnishi M, Uchiyama I, Ogura Y, Ooka T, Oshima K, Tamura A, Hattori $M$, Hayashi $\mathrm{T}$ : The whole-genome sequencing of the obligate intracellular bacterium Orientia tsutsugamushi revealed massive gene amplification during reductive genome evolution. DNA Res 2008, 15:185-199.

30. Stoeva K, Ward BF: Genome mapping of Arcobacter butzleri. FEMS Microbiol Lett 2006, 256:290-297.

31. Yasumoto K, Liu H, Jeong SM, Ohashi Y, Kakinuma S, Tanaka K, Kawamura F, Yoshikawa $\mathrm{H}$, Takahashi H: Sequence analysis of a $50 \mathrm{~kb}$ region between spoOH and $\mathrm{rrnH}$ on the Bacillus subtilis chromosome. Microbiology 1996, 142:3039-3046.

32. Huang L, Jones AME, Searle I, Patel K, Vogler H, Hubner NC, Baulcombe DC: An atypical RNA polymerase involved in RNA silencing shares small subunits with RNA polymerase II. Nat Struct Mol Biol 2008, 16:91-93.

33. Ream TS, Haag JR, Wierzbicki AT, Nicora CD, Norbeck A, Zhu JK, Hagen G, Guilfoyle TJ, Paša-Tolić L, Pikaard CS: Subunit compositions of the RNA- silencing enzymes Pol IV and Pol V reveal their origins as specialized forms of RNA Polymerase II. Mol Cell 2009, 33:1-12.

34. Karplus M, McCammon JA: Dynamics of proteins: elements and function. Annu Rev Biochem 1983, 53:263-300.

35. Karplus M, McCammon JA: Molecular dynamics simulations of biomolecules. Nat Struct Biol 2002, 9:646-652.

36. Adcock SA, McCammon JA: Molecular dynamics: survey of methods for simulating the activity of proteins. Chem Rev 2006, 106:1589-1615.

37. Pace CN, Scholtz JM: A helix propensity scale based on experimental studies of peptides and proteins. Biophys J 1998, 75:422-427.

38. López-Llano J, Campos LA, Sancho J: a-helix stabilization by alanine relative to glycine: roles of polar and apolar solvent exposures and of backbone entropy. Proteins 2006, 64:769-778.

39. Grathwohl C, Wüthrich K: The X-Pro peptide bond as an nmr probe for conformational studies of flexible linear peptides. Biopolymers 1976, 15:2025-2041.

40. Frömmel C, Preissner R: Prediction of prolyl residues in cis-conformation in protein structures on the basis of the amino acid sequence. FEBS Lett 1990, 277:159-163

41. Pal D, Chakrabarti P: Cis peptide bonds in proteins: residues involved, their conformations, interactions and locations. J Mol Biol 1999, 294:271-288.

42. Pahlke D, Freund C, Leitner D, Labudde D: Statistically significant dependence of the Xaa-Pro peptide bond conformation on secondary structure and amino acid sequence. BMC Struct Biol 2005, 5:8.

43. Hudáky I, Perczel A: Prolylproline unit in model peptides and in fragments from databases. Proteins 2008, 70:1389-1407.

44. Saha I, Chatterjee B, Shamala N, Balaram P: Crystal structures of peptide enantiomers and racemates: probing conformational diversity in heterochiral pro-pro sequences. Biopolymers 2008, 90:537-543.

45. Sosunov V, Sosunova E, Mustaev A, Bass I, Nikiforov V, Goldfarb A: Unified two-metal mechanism of RNA synthesis and degradation by RNA polymerase. EMBO J 2003, 22:2234-2244.

46. Sosunov V, Zorov S, Sosunova E, Nikolaev A, Zakeyeva I, Bass I, Goldfarb A, Nikiforov $V$, Severinov K, Mustaev A: The involvement of the aspartate triad of the active center in all catalytic activities of multisubunit RNA polymerase. Nucleic Acids Res 2005, 33:4202-4211.

47. Castro C, Smidansky E, Maksimchuk KR, Arnold JJ, Korneeva VS, Götte M, Konigsberg W, Cameron CE: Two proton transfers in the transition state for nucleotidyl transfer catalyzed by RNA- and DNA-dependent RNA and DNA polymerases. Proc Natl Acad Sci USA 2007, 104:4267-4272.

48. Huang Y, Beaudry A, McSwiggen J, Sousa R: Determinants of ribose specificity in RNA polymerization: effects of $\mathrm{Mn}^{2+}$ and deoxynucleoside monophosphate incorporation into transcripts. Biochemistry 1997, 36:13718-13728.

49. Mason PB, Struhl K: Distinction and relationship between elongation rate and processivity of RNA Polymerase II in vivo. Mol Cell 2005, 17:831-840.

50. Feig M, Burton ZF: RNA polymerase II flexibility during translocation from normal mode analysis. Proteins 2010, 78:434-46.

51. Seibold SA, Singh BN, Zhang C, Kireeva M, Domecq C, Bouchard A, Nazione AM, Feig M, Cukier Rl, Coulombe B, Kashlev M, Hampsey M Burton ZF: Conformational coupling, bridge helix dynamics and active site dehydration in catalysis by RNA polymerase. Biochim Biophys Acto 2010, 1799:575-587.

52. Huang $X$, Wang D, Weiss DR, Bushnell DA, Kornberg RD, Levitt M: RNA polymerase II trigger loop residues stabilize and position the incoming nucleotide triphosphate in transcription. Proc Natl Acad Sci USA 2010, 107:15745-15750.

53. Yildirim $Y$, Doruker $P$ : Collective motions of RNA polymerases. Analysis of core enzyme, elongation complex and holoenzyme. J Biomol Struct Dyn 2004, 22:267-280.

54. Toulokhonov I, Zhang J, Palangat $M$, Landick R: A central role of the RNA polymerase trigger loop in active-site rearrangement during transcriptional pausing. Mol Cell 2007, 27:406-419.

55. Tavormina PL, Reznikoff WS, Gross CA: Identifying interacting regions in the $\beta$ subunit of Escherichia coli RNA polymerase. J Mol Biol 1996, 258:213-223.

56. Cunningham BC, Wells JA: High-resolution epitope mapping of hGHreceptor interactions by alanine-scanning mutagenesis. Science 1989, 244:1081-1085

57. O'Malley MA, Elliott KC, Haufe C, Burian RM: Philosophies of funding. Cell 2009, 138:611-615. 
58. Wiesler SC, Weinzierl ROJ: The Linker domain of basal transcription factor TFIIB controls distinct recruitment and transcription stimulation functions. Nucl Acids Res 2010.

59. Van Der Spoel D, Lindahl E, Hess B, Groenhof G, Mark AE, Berendsen HJ: GROMACS: fast, flexible, and free. J Comput Chem 2005, 26:1701-1718.

60. Arnold K, Bordoli L, Kopp J, Schwede T: The SWISS-MODEL workspace: a web-based environment for protein structure homology modelling. Bioinformatics 2006, 22:195-201.

61. Heinig M, Frishman D: STRIDE: a web server for secondary structure assignment from known atomic coordinates of proteins. Nucleic Acids Res 2004, 32 Web Server: W500-502.

62. Humphrey W, Dalke A, Schulten K: VMD: visual molecular dynamics. J Mol Graph 1996, 14:33-38.

63. Bern M, Goldberg D: Automatic selection of representative proteins for bacterial phylogeny. BMC Evol Biol 2005, 5:34.

64. Berkholz DS, Krenesky PB, Davidson JR, Karplus PA: Protein Geometry Database: a flexible engine to explore backbone conformations and their relationships to covalent geometry. Nucleic Acids Res 2010, 38: D320-325.

doi:10.1186/1741-7007-8-134

Cite this article as: Weinzierl: The nucleotide addition cycle of RNA polymerase is controlled by two molecular hinges in the Bridge Helix domain. BMC Biology 2010 8:134.

\section{Submit your next manuscript to BioMed Central} and take full advantage of:

- Convenient online submission

- Thorough peer review

- No space constraints or color figure charges

- Immediate publication on acceptance

- Inclusion in PubMed, CAS, Scopus and Google Scholar

- Research which is freely available for redistribution

Submit your manuscript at www.biomedcentral.com/submit 